\title{
A curved array photoacoustic tomography system for small animal imaging
}

Andres Aguirre, John Gamelin, Anastasios Maurudis, Fei Huang, Diego Castillo, et al.

Andres Aguirre, John Gamelin, Anastasios Maurudis, Fei Huang, Diego Castillo, Puyun Guo, Lihong V. Wang, Quing Zhu, "A curved array photoacoustic tomography system for small animal imaging," Proc. SPIE 6437, Photons Plus Ultrasound: Imaging and Sensing 2007: The Eighth Conference on Biomedical Thermoacoustics, Optoacoustics, and Acoustooptics, 64370V (13 February 2007); doi: 10.1117/12.707548

SPIE. Event: SPIE BiOS, 2007, San Jose, California, United States 


\title{
A curved array photoacoustic tomography system for small animal imaging
}

\author{
Andres Aguirre ${ }^{\mathrm{a}}$, John Gamelin ${ }^{\mathrm{a}}$, Anastasios Maurudis ${ }^{\mathrm{a}}$, Fei Huang ${ }^{\mathrm{a}}$, Diego Castillo ${ }^{\mathrm{a}}$, Puyun Guo ${ }^{\mathrm{a}}$, \\ Lihong V. Wang ${ }^{\mathrm{b}}$, and Quing $\mathrm{Zhu}^{\mathrm{a}}$ \\ ${ }^{a}$ Dept. of Electrical Engineering, University of Connecticut, Storrs-Mansfield, CT 06269 \\ ${ }^{b}$ Dept. of Biomedical Engineering at Washington University in St. Louis, St. Louis, MO 63130
}

\begin{abstract}
We have developed and tested a photoacoustic imaging system based on a 128 element curved-phased ultrasonic array, which spans a quarter of a complete circle with a radius of curvature equal to $25 \mathrm{~mm}$. The center frequency of the array is $5 \mathrm{MHz}$ with $60 \%$ bandwidth. The physical dimensions of the elements are 10x0.3mm (elevation $\mathrm{x}$ azimuth) with an elevation focus of $19 \mathrm{~mm}$. Earlier we reported acoustic measurements of the axial and lateral resolutions of the system that were limited by the impulse response of the narrowband source used in the test. In this paper we discuss photoacoustic characterization of the system including resolution and sensitivity. The array forms the building block for a 512-element ring designed for complete tomographic imaging of small animals. Imaging results of phantoms will be compared with simulations.
\end{abstract}

Keywords: Photoacoustic, optoacoustic, thermoacoustic, tomography, imaging.

\section{INTRODUCTION}

It has been shown that tumors possess enhanced optical absorption contrast compared to healthy tissue, when imaged with near infrared light ${ }^{1}$. However, light is highly scattered in biological tissues. In consequence, pure optical imaging techniques, like diffuse optical tomography (DOT) and optical coherence tomography (OCT), suffer from limited resolution (DOT) or limited imaging depth (OCT). On the other hand, ultrasound scattering is 2-3 orders of magnitude weaker than optical scattering in biological tissue. As a result, ultrasound imaging can provide good resolution. However, it has weak contrast for early stage tumors, as it is based on the detection of mechanical properties in biological tissue ${ }^{2}$.

Photoacoustic imaging is an emerging technique, in which a short-pulsed laser beam penetrates into the tissue sample diffusively ${ }^{3-9}$. Thermoelastic expansion resulting from a transient temperature rise, on the order of $10 \mathrm{mK}$, caused by the laser irradiation, generates photoacoustic waves that can be measured around the sample by wide-band ultrasound transducers. The acquired photoacoustic waves are used to reconstruct, at ultrasound resolution, the optical absorption distribution. The resolution and penetration can be matched to the application through the ultrasound frequency. Studies using tissue phantoms ${ }^{10}$ show that photo-acoustic imaging has the capability to image heterogeneous tissue structures with sub-millimeter resolution at depths up to several centimeters.

Currently there are two popular approaches for performing photoacoustic imaging. One uses a single wideband point detector or a single focused transducer and scanning it around the area of interest. This approach requires the actual physical movement of the detector or sample. The second one involves ultrasound transducer arrays. This approach does not require physical movement of the transducer or sample. However, the electronics involved with these systems is much more complex. In clinical applications, data acquisition speed is a very important factor influencing the quality and validity of imaging. A fast data acquisition approach minimizes the effects due to movement of the patient. As a result, ultrasound transducer arrays are more suitable for clinical applications as the acquisition time is much less than that of using a single detector approach.

We have developed a photoacoustic system using an ultrasound transducer array in a quarter circle configuration for imaging of small animals. Previously, we reported results of extensive measurements of acoustic and electronic characteristics of our detection system ${ }^{11-12}$. These results provide deep understanding regarding the performance of our detection system. In this paper we report the results from a series of photoacoustic tests that have

Photons Plus Ultrasound: Imaging and Sensing 2007: The Eighth Conference on Biomedical Thermoacoustics, Optoacoustics, and Acousto-optics, edited by Alexander A. Oraevsky, Lihong V. Wang, Proc. of SPIE Vol. 6437, 64370V, (2007) · 1605-7422/07/\$18 · doi: 10.1117/12.707548 
been performed on our system to better understand its capabilities. This system represents a building block for a full circle system for photoacoustic tomography.

\section{SYSTEM DESCRIPTION}

The overall block diagram of our photoacoustic imaging system is shown in Figure 1. It consists of a fixed ultrasound transducer array mounted on a water tank and connected to a signal processing circuitry. A data acquisition system is used to digitize and store the received signals. A pulsed laser illuminates the sample and triggers the data acquisition.

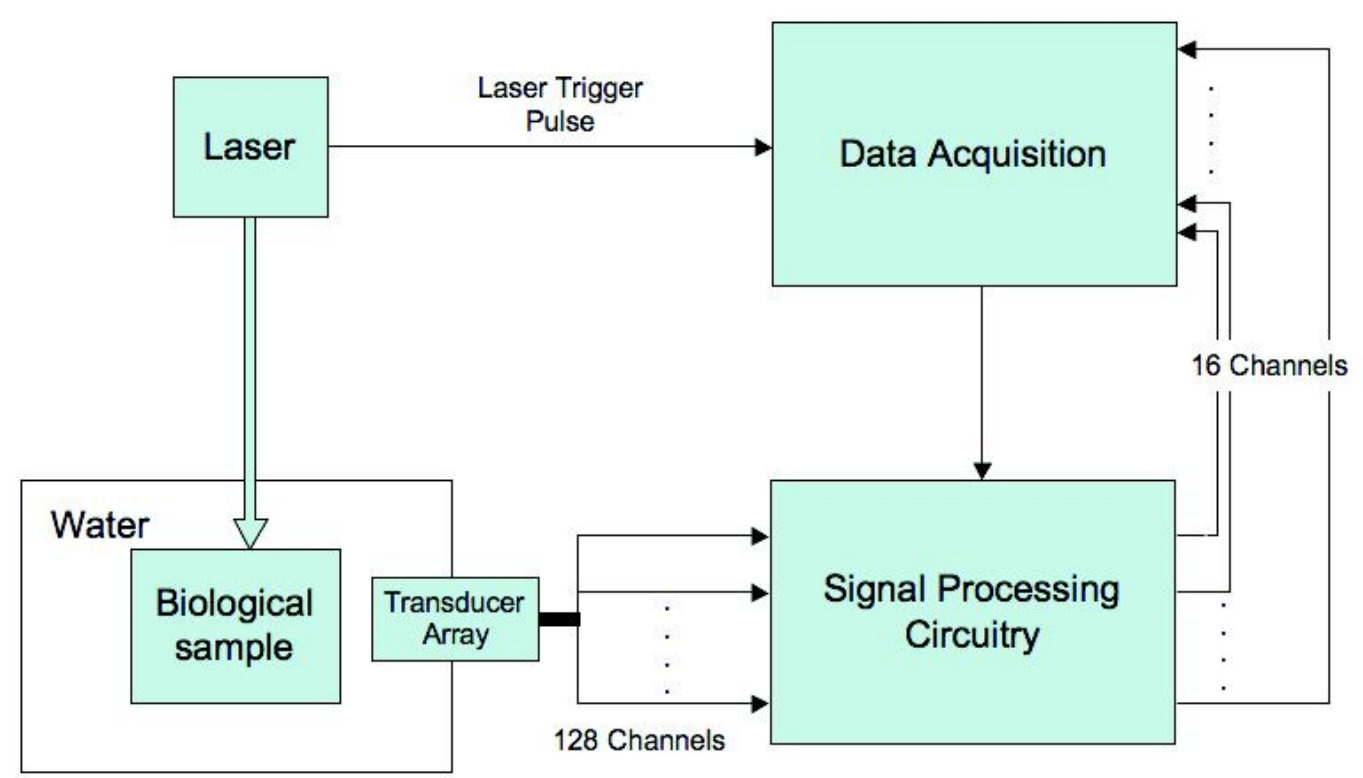

Figure 1. Block diagram of the photoacoustic imaging system.

The transducer array was custom manufactured by Imasonic (Besancon, France) to our specifications. It consists of 128 elements in a quarter circle aperture with a radius of curvature equal to $25 \mathrm{~mm}$. The center frequency of the transducer array is $5 \mathrm{MHz}$ with a bandwidth greater than $60 \%$. The physical dimensions of each element are $10 \mathrm{~mm}$ and $0.208 \mathrm{~mm}$ in elevation and lateral aperture respectively. The inter-element spacing is $0.1 \mathrm{~mm}$. Each of the elements is unfocused in the lateral direction, but focused in the elevation plane. The elevation focal plane is located at the midpoint of the elevation aperture with a focal depth equal to $19 \mathrm{~mm}$. The combined focal points of all the elements form a quarter circle with a radius of curvature of $6 \mathrm{~mm}$. The array elements are made of a piezocomposite material, which allows direct shaping to accomplish the elevation focusing without an acoustic lens. Piezocomposite materials have a high coupling coefficient that confers to the transducers a high sensitivity and signal to noise ratio $(+10 \mathrm{~dB}$ to $+30 \mathrm{~dB}$ compared to conventional technology). The intrinsic damping of this material allows a high bandwidth, while keeping a good sensitivity.

The laser system used in our setup consists of a Q-switch pulsed Nd:YAG laser from Symphotic Tii Corp. (Camarillo, CA) running at a pulse repetition rate of $15 \mathrm{~Hz}$ and pulse duration of $12 \mathrm{~ns}$. The Nd:YAG laser is used to pump a variable wavelength $(700-1000 \mathrm{~nm})$ Ti:Sapphire laser also from Symphotic Tii Corp. The energy level out of the Ti:Sapphire laser has been controlled to less than $50 \mathrm{~mJ}$. To homogenize and expand the laser beam to a diameter close to 2 inches, an engineered diffuser (ED1-C20) from Thorlabs has been used.

The other components of the system, the signal processing circuitry and the data acquisition system, have been designed and developed in our lab. The data acquisition system consists of 4 standard PCI half-length cards that can be installed on any PC computer running Microsoft Windows. Each card has 4 channels with a sampling rate of $40 \mathrm{MHz}$ and 12 bits of quantization and uses asynchronous static random access memory (ASRAM). In total the system has 16 DAQ channels available. 
Due to the limited number of DAQ channels, compared to the number of elements of the ultrasound transducer array, the signal processing circuitry was designed to implement an 8:1 multiplexing modality as shown in Figure 2. The input signals are first processed by a high-gain and low-noise amplifier (LNA), AD8099. An eight input to one output multiplexer (MUX), MAX4051, feeds one of the eight LNA output signals to our variable gain amplifier (VGA), AD604. The control signals from the backplane consist of the three address lines used to set the multiplexer as well as the VGA. The LNA amplifiers have a disable feature that allows them to be placed in a high impedance state when it is not activated. Thus, only one of the eight LNA amplifiers is enabled at any given laser firing session. The off-isolation of a disabled LNA amplifier is -60 decibel, which greatly minimizes the crosstalk among the channels. Finally the output of the VGA is passed to data acquisition system through coaxial cable.

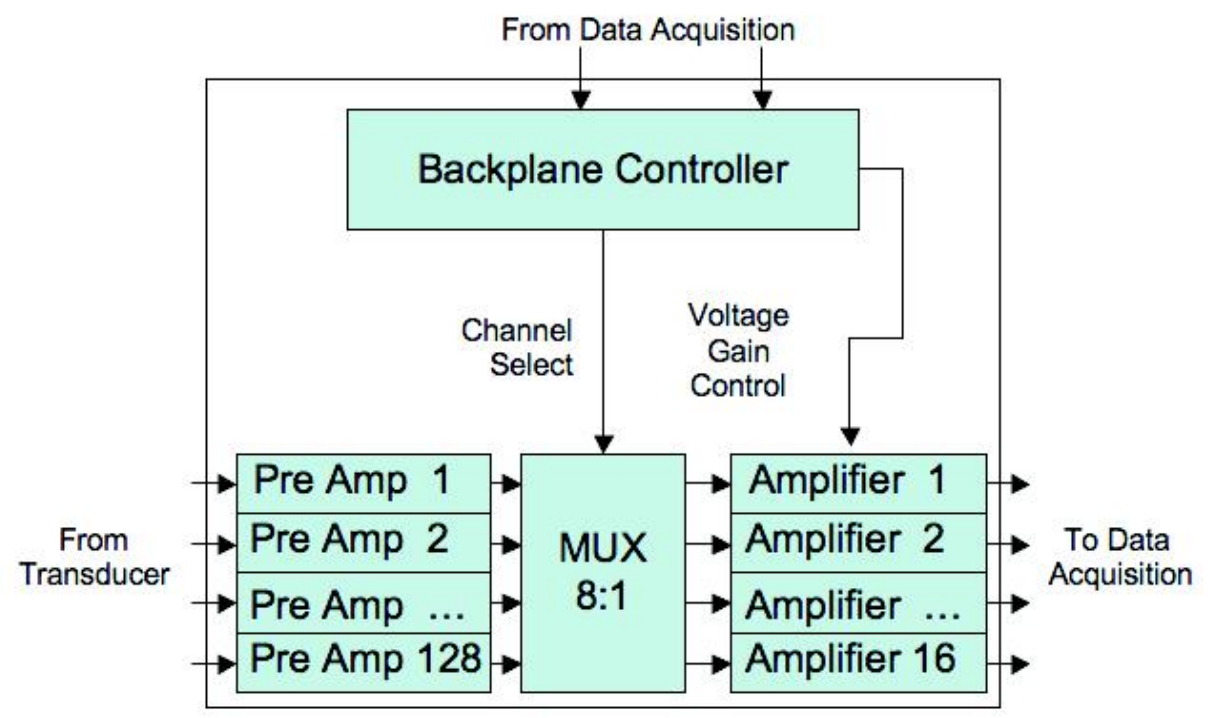

Figure 2. Signal processing circuitry block diagram

As shown in previously reported measurements ${ }^{11-12}$, the frequency response of the signal processing circuitry is relatively flat within our bandwidth of interest $(2 \mathrm{MHz}-8 \mathrm{MHz})$. The measured $\mathrm{SNR}$ is $95 \mathrm{~dB}, 75 \mathrm{~dB}$, and $55 \mathrm{~dB}$ for gains of $54 \mathrm{~dB}, 74 \mathrm{~dB}, 94 \mathrm{~dB}$, respectively. Measurable crosstalk only appeared at the output for an input signal level of $20 \mathrm{dBm}$. However, the input level is not expected to approach $-20 \mathrm{dBm}$. Thus, this design is good for crosstalk compression. Both frequency responses and crosstalk were measured with a network analyzer.

\section{SYSTEM CHARACTERIZATION}

Before moving on to actual experiments with animals, first the system had to be characterized to better understand its capabilities. In this section, we show a series of experiments that were performed to characterize our photoacoustic system. Simulations, using Field II, are also shown as a verification of the experimental results. Field II is an ultrasound simulation program that allows the calculation of the received response from point sources by acoustic reciprocity. The simulated signals were then input into our delay and sum reconstruction algorithm for processing and image formation.

The first step in our characterization was to look at the photoacoustic signal generated by the front face of one of the elements of the transducer. The result is shown in Figure 3a. This signal represents the impulse response of the elements of the ultrasound transducer. By applying the Fast Fourier Transform to the measured impulse response, the impulse response in frequency domain can be obtained as shown in figure $3 \mathrm{~b}$. 


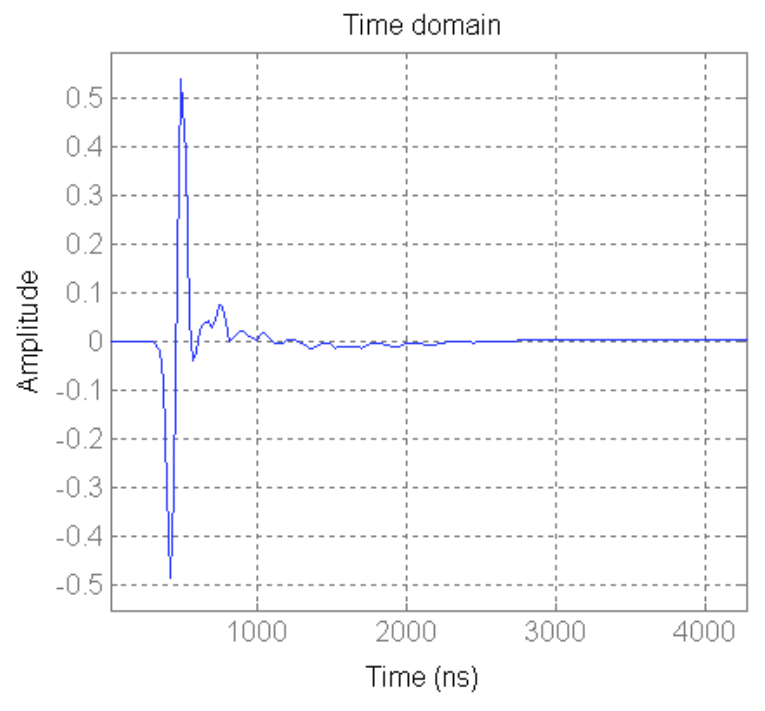

a) Time domain.

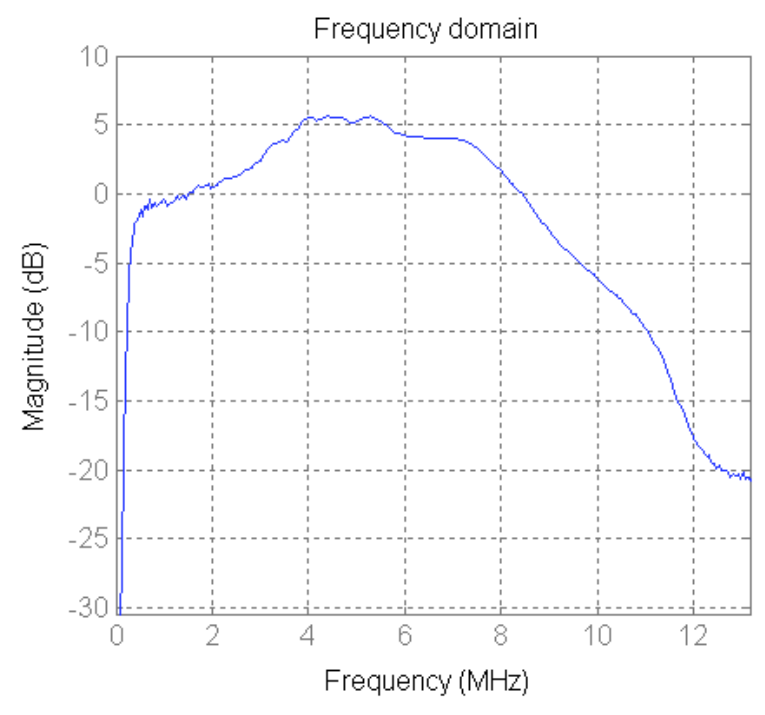

b) Frequency domain

Figure 3. Measured impulse response.

From the frequency domain impulse response it can be observed that the center frequency of the transducer is at 5.1 MHz. Additionally, the points of $-6 \mathrm{~dB}$ can be identified at approximately $2.10 \mathrm{MHz}$ and $8.28 \mathrm{MHz}$. These results are in agreement with the specified center frequency and bandwidth for the ultrasound transducer array. Additionally, by looking at the upper point of $-3 \mathrm{~dB}$, the axial resolution of the transducer can be estimated ${ }^{13}$ by applying:

$$
\delta_{\min } \approx c / f_{\max }
$$

where $f_{\max }$ in our system is approximately $7.7 \mathrm{MHz}$. The calculated resolution from the impulse response for our system is equal to $190 \mu \mathrm{m}$.

To further evaluate the resolution of our system, we can also look at the measured and simulated point spread functions (PSF). Setting up a point absorber in Field II we were able to simulate the point-spread function of the system. The point absorber was placed at the elevation focus of the transducer array, which according to the specifications is located at $6 \mathrm{~mm}$, in the axial direction, before the center of curvature of the ultrasound array and in the center of the aperture in the lateral direction as shown in Figure 4a.

After processing the simulated point spread function with a delay and sum algorithm, the reconstructed image shows a well-defined point at the specified location as shown in Figure 4b. From measurement of the beam profiles along the lateral and axial axis at the location of the point absorber the simulated resolution of the system is about 170 $\mu \mathrm{m}$ in the lateral direction and $150 \mu \mathrm{m}$ in axial direction. The resolution here is defined as the distance between the $-6 \mathrm{~dB}$ points.

Experimentally, the point-spread function can be measured using a very small absorber like black thread with a diameter of $80 \mu \mathrm{m}$. To allow the light to properly hit the thread, which had been stretched between two plastic holders, it had to be positioned with an angle of approximately $20^{\circ}$ in the axial direction as shown in Figure 5a. The reconstructed image, as well as the lateral and axial beam profiles, is shown in Figures 5b, 5c, and 5d respectively. From the results the measured distance between the points of $-6 \mathrm{~dB}$ is equal to $250 \mu \mathrm{m}$ and $260 \mu \mathrm{m}$ in the lateral and axial directions. In order to estimate the effective measured resolution, the size of the thread has to be deconvolved (e.g. subtracted) from the measured distance. After correction, the measured lateral resolution is equal to $170 \mu \mathrm{m}$ and the axial resolution is equal to $180 \mu \mathrm{m}$. These measured values of the resolution are in concordance with the simulated values 


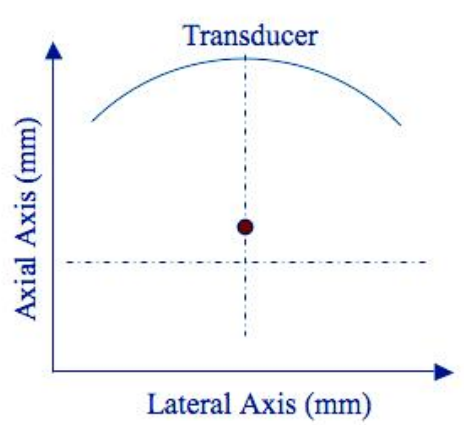

a) Simulation Arrangement.

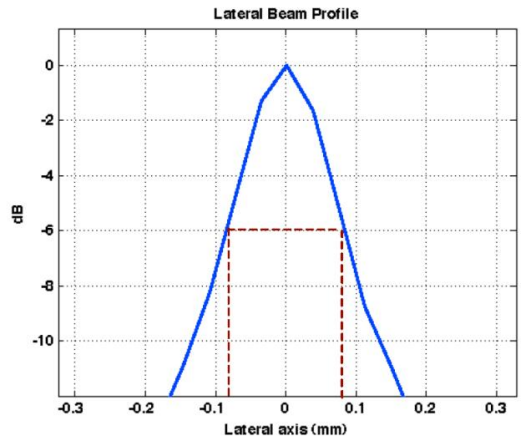

c) Lateral Beam Profile

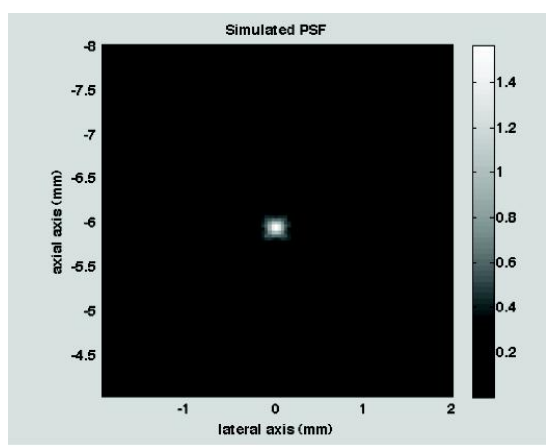

b) Reconstructed Image.

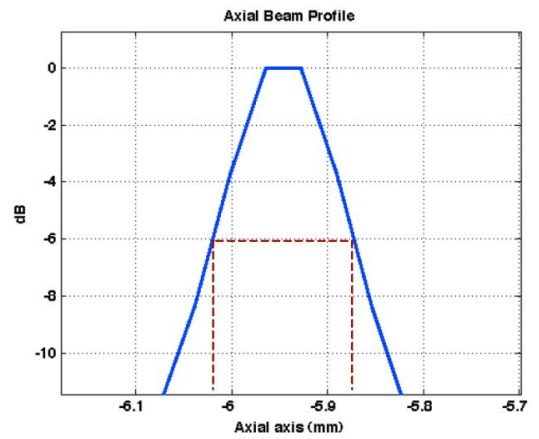

d) Axial Beam Profile

Figure 4. Simulated PSF.

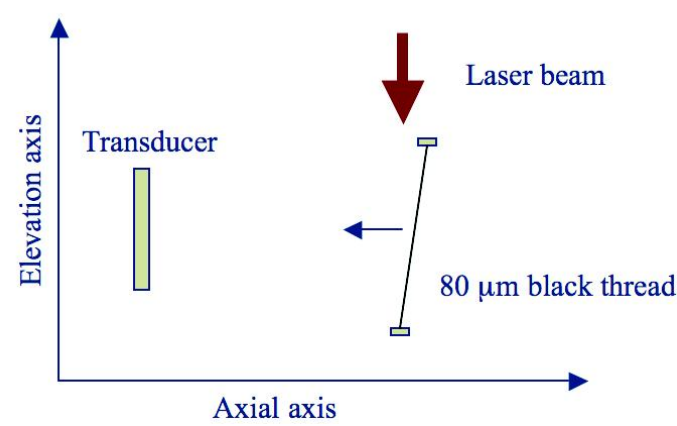

a) Measurement Setup.

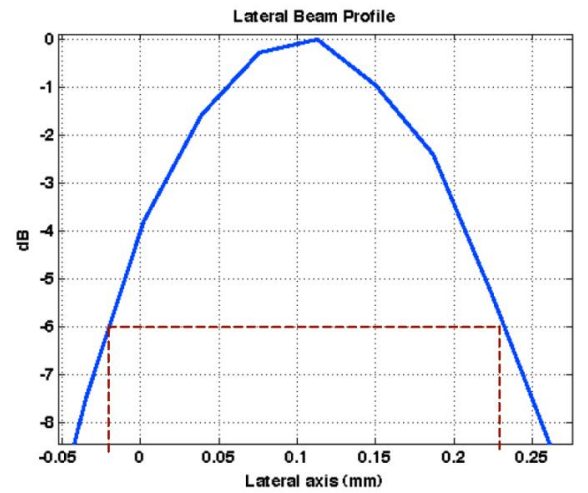

c) Lateral Beam Profile

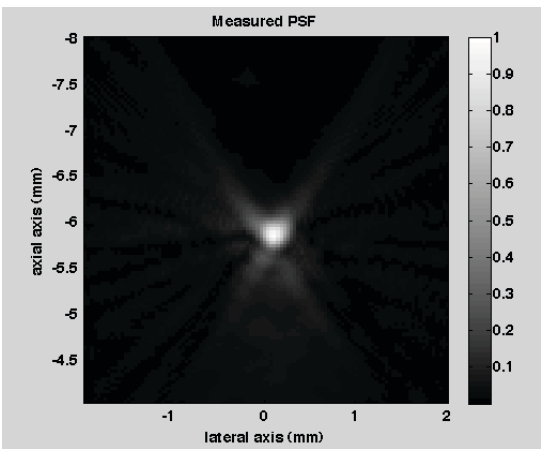

b) Reconstructed Image.

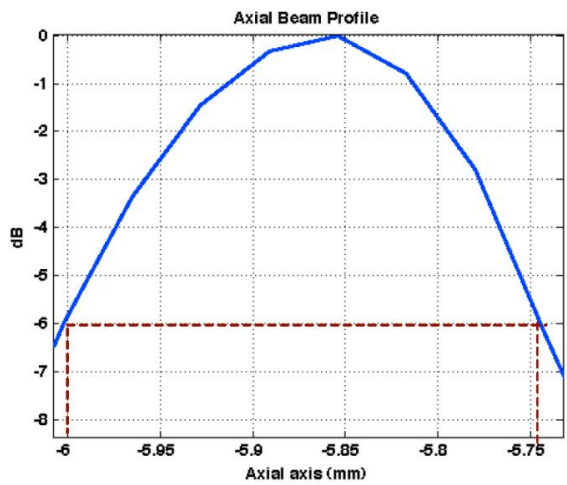

d) Axial Beam Profile

Figure 5. Measured PSF. 
An additional experiment to further verify the resolution of the system was performed using two identical threads such as those used in the PSF measurement. The threads were placed with a tapered separation as shown in Figure 6a, which shows the view of the setup as seen from the transducer in the axial direction. The height of the threads was adjusted to obtain the desired separation of the threads at the mid-elevation plane. Also, as in the previous case the sample had to b placed with an angle of approximately $20^{\circ}$ in the axial direction. The results show that at the point where the threads are separated approximately $250 \mu \mathrm{m}$ the system is still able to clearly resolve each of them as shown in Figure $6 \mathrm{~b}$. This is seen more clearly in the profile plot of Figure $6 \mathrm{c}$.

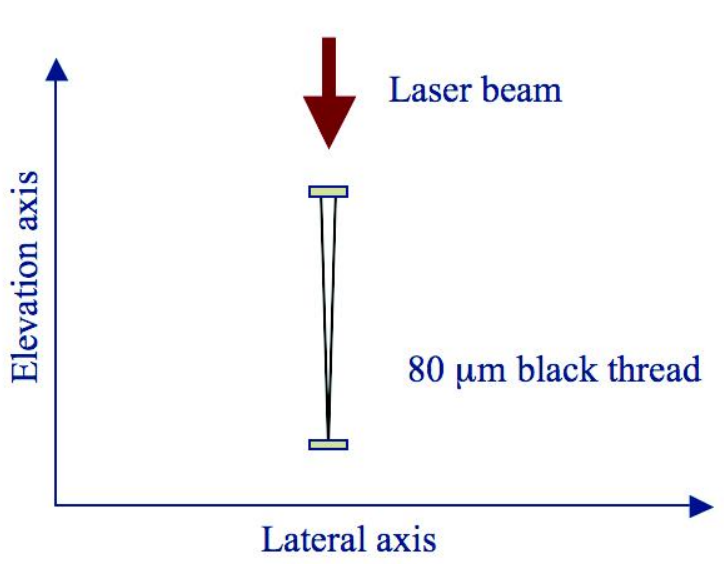

a) Setup of Measurement

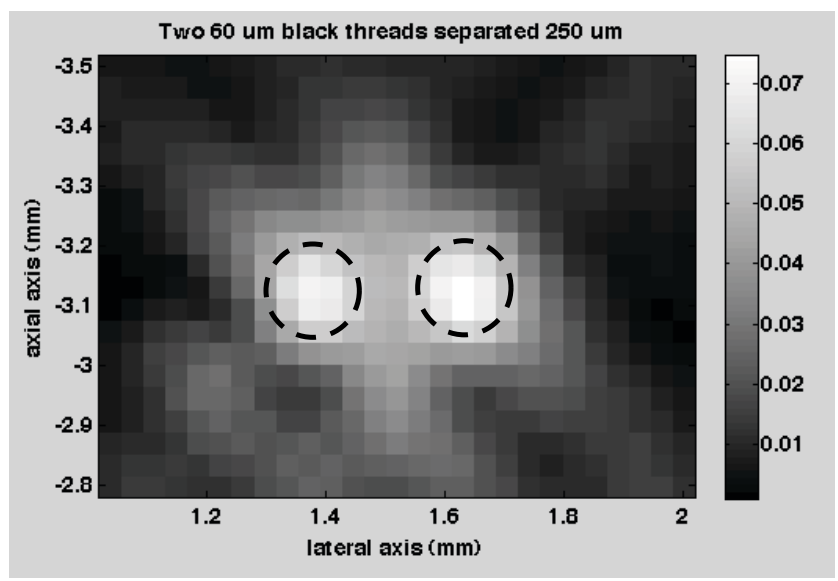

b) Reconstructed Image

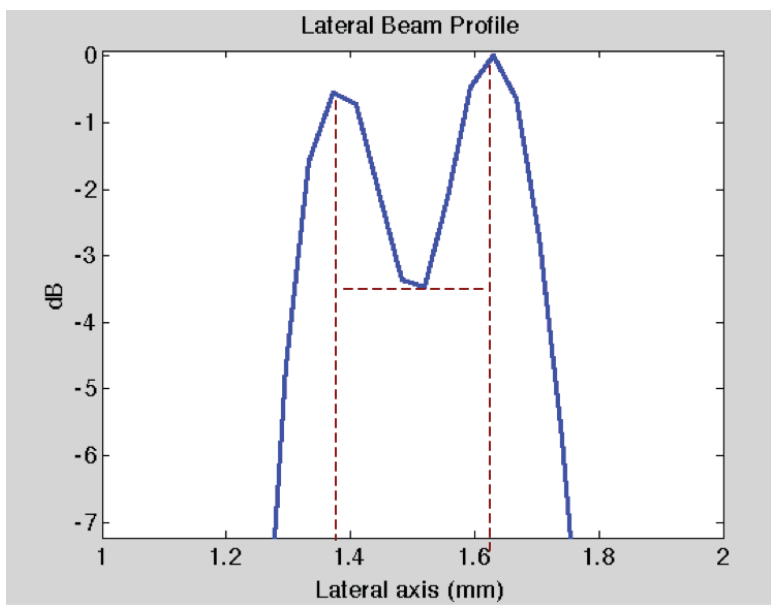

c) Lateral Beam Profile

Figure 6. Resolution measurement.

Another important characterization is the measurement of the relative sensitivity within the field of view of the transducer array as function of the position. The relative sensitivity was measured using a small piece of pencil lead (approximately $0.5 \mathrm{~mm}$ in diameter and $1.5 \mathrm{~mm}$ in length) mounted on a non-absorbing fiber. By moving the pencil lead through a representative area, we were able to obtain reconstructed images at each point. The values at each location were normalized to the peak and plotted across the measurement domain. The scan region spanned from $-17 \mathrm{~mm}$ to +2 $\mathrm{mm}$ in the axial axis and from $+11 \mathrm{~mm}$ to $-11 \mathrm{~mm}$ in the lateral axis respect to the center of curvature of the array. The steps used between each measurement were $1 \mathrm{~mm}$ when moving axially at the lateral center and when moving laterally at the elevation focus distance in the axial direction. For the rest of the field the measurements were performed every 2 mm both laterally and axially. The results, as seen on Figures $7 \mathrm{~b}$ and $7 \mathrm{c}$, show, as expected, that the sensitivity across the 
field of view is not uniform and that it peaks close to the elevation focus and extends up to the center of curvature where it starts to fall again.

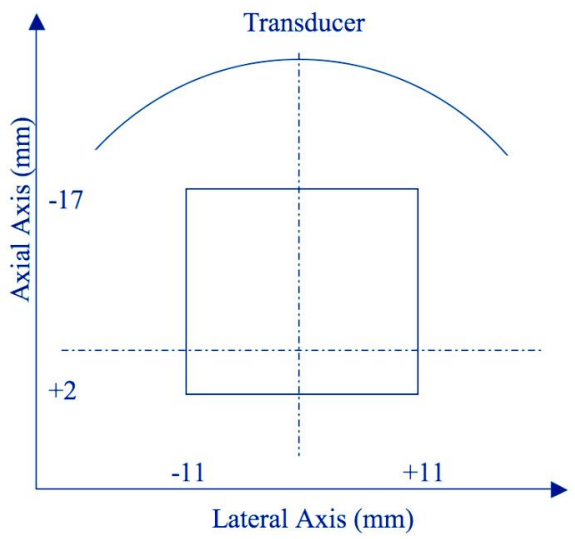

a) Measurement Setup

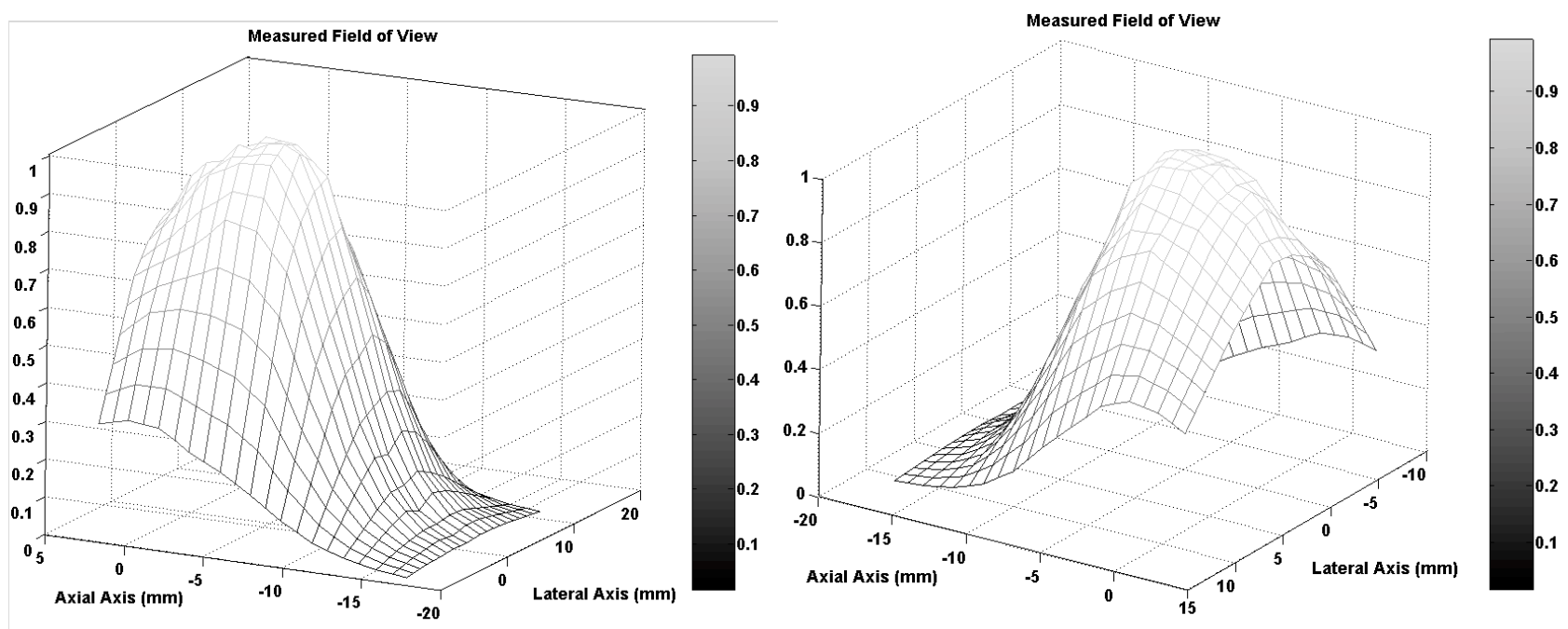

b) Measured Field of View

Figure 7. Field of view measurements.

Additionally, the measured sensitivity across the field of view can be compared with that obtained via simulation. For the simulation, a grid of point absorbers was created in Field II, where each point is spaced $1 \mathrm{~mm}$ in both lateral and axial direction. The sensitivity plot was then generated by selection of the peak absorption values at each source location from the reconstructed image. Figure 8 presents, both simulated and measured, plots for the lateral and axial profiles of the field of view. The results demonstrate good agreement between the simulated and measured profiles.

As a preliminary step toward imaging small animals, the system was tested using a more realistic sample, such as a small polyethylene tube filled with blood. The inner diameter of the tube was $0.58 \mathrm{~mm}$, making it an appropriate sample to emulate a blood vessel. The resulting image, presented in Figure 9, demonstrates the ability of the system to successfully reconstruct the blood tube with the correct dimensions. It is important to note that this image was obtained without expanding the beam. For that reason the length of the reconstructed tube was limited by the size of the laser beam, which at the time was just over $10 \mathrm{~mm}$ in diameter. The artifacts shadowing the tube may be due to reflections stemming from acoustic impedance mismatches at the tube walls. 


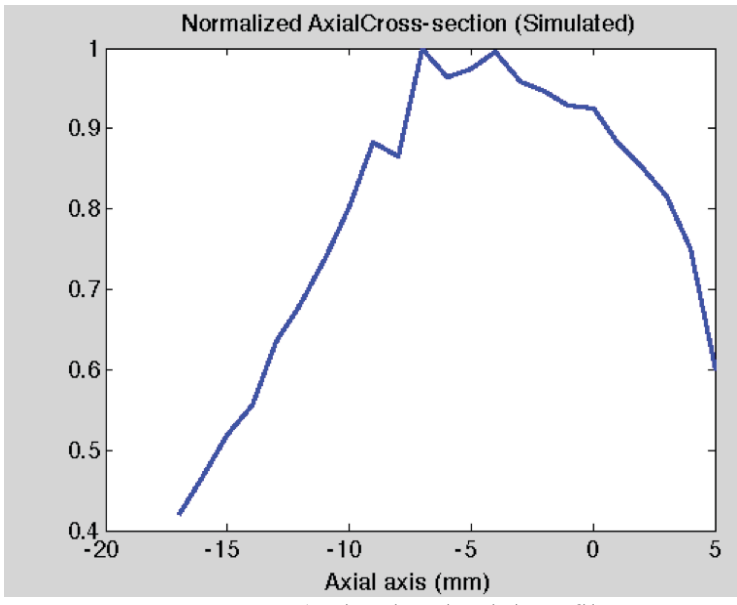

a) Simulated axial profile.

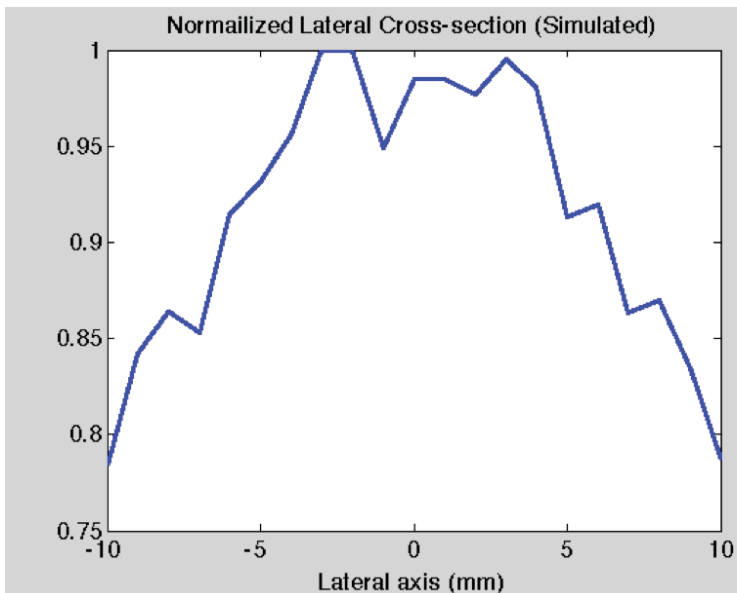

c) Simulated lateral profile.

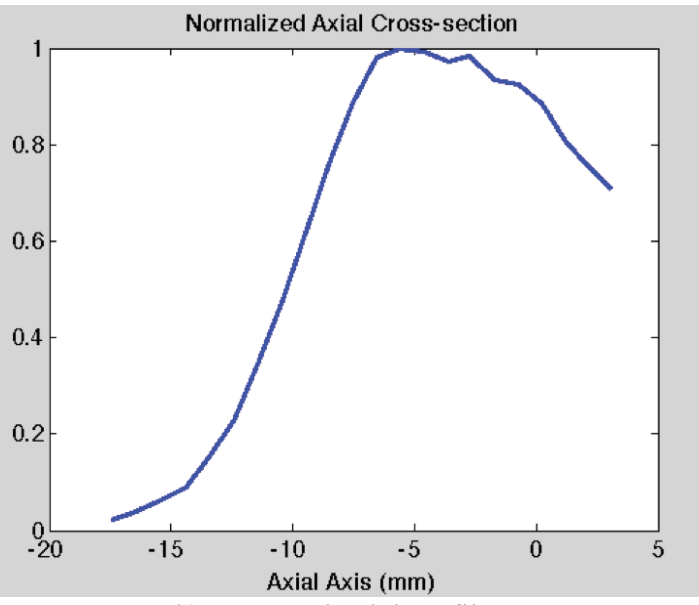

b) Measured axial profile.

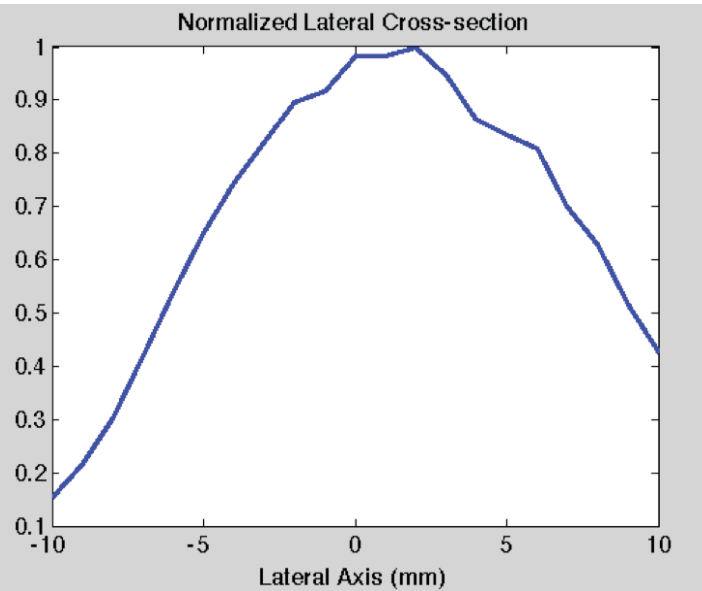

d) Measured lateral profile.

Figure 8. Field of View.

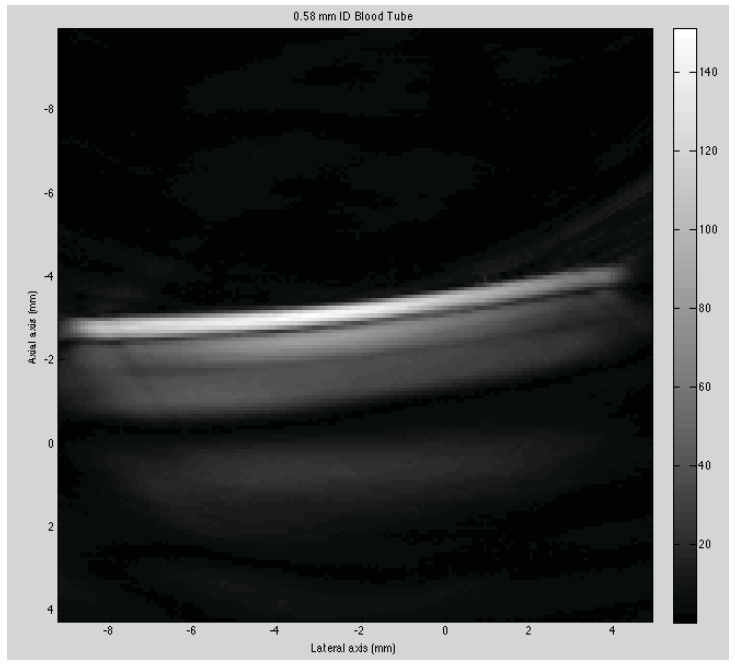

Figure 9. Polyethylene tube filled with blood. 


\section{CONCLUSION}

We have developed a photoacoustic imaging system for small animals, using a curved ultrasound transducer array. The resolution of the system has been measured to be in the order of $200 \mu \mathrm{m}$, both in the lateral and axial directions. The sensitivity of the system has been shown to peak from a few millimeters before the elevation focus to the center of the array, which creates a fairly uniform imaging region at the center of the array. A preliminary image of a biological sample was successfully obtained using a polyethylene tube filled with blood. The system will be used for imaging of tumors in mice and expanded to the full 512-element tomographic configuration.

\section{ACKNOWLEDGMENTS}

We acknowledge support from NIH R01 NS46214. The author can be contacted at (860) 486-3673 or via e-mail at andres@engr.uconn.edu.

\section{REFERENCES}

1. Manohar, S., A. Kharine, Alexei Kharine, Johan C. G. van Hespen, Wiendelt Steenbergen, Ton G. van Leeuwen Photoacoustic mammography laboratory prototype: imaging of breast tissue phantoms. J Biomed Opt. 2004 9(6): 1172-81.

2. L. V. Wang, Ultrasound-mediated biophotonic imaging: a review of acousto-optical tomography and photoacoustic tomography, Dis Markers, vol. 19, pp. 123-38, 2003.

3. Kruger RA, Liu P, Fang YR, and Appledorn CR. Photoacoustic ultrasound (PAUS)-reconstruction tomography. Med. Phys., vol. 22, pp. 1605-1609. October 1995.

4. Wang XD, Pang YJ, Ku G, Xie XY, Stoica G. and Wang LV. Noninvasive laser-induced photoacoustic tomography for structural and functional in vivo imaging of the brain. Nat. Biotechnol., vol. 21, pp. 803-806, July 2003.

5. Andreev VG, Karabutov AA, Oraevsky AA. Detection of ultrawide-band ultrasound pulses in optoacoustic tomography. IEEE Trans Ultrason Ferroelectr Freq Control. 2003 Oct;50(10):1383-90.

6. Esenaliev RO, Karabutov AA, Oraevsky AA. Sensitivity of laser opto-acoustic imaging in detection of small deeply embedded tumors. IEEE Journal of selected topics in Quantum Electronics 5, 981-988,1999

7. Wang X, Xie X, Ku G, Wang LV, Stoica G. Noninvasive imaging of hemoglobin concentration and oxygenation in the rat brain using high-resolution photoacoustic tomography. J Biomed Opt. 2006 MarApr;11(2):24015.

8. Ku G, Fornage BD, Jin X, Xu M, Hunt KK, Wang LV. Thermoacoustic and photoacoustic tomography of thick biological tissues toward breast imaging. Technol Cancer Res Treat. 2005 Oct;4(5):559-66.

9. Viator J.A, Au G., Paltauf G, Jacques SL, Prahl SA, Ren HW, Chen ZP and Nelson JS. Clinical testing of s photoacoustic probe for port wine stain depth determination. Lasers in Surgery and Medicine 30,141-148, 2002.

10. S.A. Carp, A. Guerra, S.Q. Duque, and V. Venugopalan, Optoacoustic imaging using interferometric measurement of surface displacement. App. Phy. Letters, vol. 85, pp.5772-5774, December 2004.

11. Anastasios Murudis, F. H., Diego Castillo, Puyun Guo, Shikui Yan, Quing Zhu. A novel electronic architecture used to support biomedical photo-acoustic imaging. IEEE International Symposium on Circuits and Systems, 2006.

12. Maurudis A, Huang F, Guo P, Yan SK, Castillo D, Wang LV, Zhu Q. A photoacoustic imaging system employing a curved-phased ultrasonic array and parallel electronics. SPIE Proceedings, Vol 6086, 60861Q-1:11, 
2006.

13. Sergey A. Ermilov, Andre Conjusteau, Ketan Mehta, Ron Lacewell, P. Mark Henrichs, and Alexander A. Oraevsky. SPIE Proceedings, Vol 6086, 608609-1:12, 2006. 